\title{
Modelos Estocásticos De Elección De Compra Recurrente Aplicados En Hogares De La Ciudad De México
}

\author{
Laura Fischer \\ Facultad de Contaduría y Administración UNAM, Mexico \\ Jorge Espejo \\ Universidad Autónoma del Estado de Morelos, Mexico
}

Doi: 10.19044/esj.2018.v14n5p86 URL:http://dx.doi.org/10.19044/esj.2018.v14n5p86

\begin{abstract}
This paper focuses on examining the behaviors of repeated purchase, brand duplication, shared loyalty, and double risk using stochastic models of recurring purchase decision. A quantitative study was carried out using the panel technique in 60 homes in Mexico City. Within a period of 12 weeks, the recurring purchasing behavior of these households was recorded and analyzed. The results show, in a scientific way, the behavior of recurrent purchases in Mexican households.
\end{abstract}

Keywords: Repetitive purchasing behavior, brand duplicity, shared brand loyalty, double risk, stochastic analysis

\section{Resumen}

Con el objetivo de examinar las conductas de compra repetida, duplicación de marca, lealtad compartida y doble riesgo utilizando modelos estocásticos de elección de compra recurrente. Se realizó un estudio cuantitativo mediante la técnica de panel en 60 hogares de la ciudad de México; durante un periodo de 12 semanas se registraron y analizaron los comportamientos de compra recurrente de esos hogares. Los resultados demuestran de manera científica el comportamiento de compra recurrente en hogares mexicanos

Palabras-claves: Comportamiento de compra repetitivo, duplicidad de marca, lealtad de marca compartida, doble riesgo, análisis estocástico

\section{Introducción}

Cuando un consumidor realiza una compra toma varias decisiones simultáneas: qué comprar, cuándo comprar, cómo comprar y cuánto comprar; 
cuando realiza compras repetitivas y estas son a las mismas marcas se considera que existe una lealtad; la lealtad es considerada uno de los factores más importantes para explicar el sentido de pertenencia y cariño del consumidor por una marca,

Una cuestión permanente en investigadores y académicos de la mercadotecnia sobre el comportamiento de elección de marcas es si ese comportamiento es estocástico o si las causas y explicaciones son relativas a todo el comportamiento humano; múltiples investigaciones sobre la conducta del consumidor muestra evidencia de que la elección de marca es sustancialmente un comportamiento estocástico; destacando los trabajos de Howard (1971), Rivas (1981), Cebollada (1996), Dekimpe, Steenkamp, Mellens \& Abeele (1997), Orta (2001), Ballester (2004), Yunda (2009), Vidal, Londoño \& Contreras (2011), Sánchez \& Hasbleidy (2014), Valbuena, (2016).

Mordecki (2010) Sánchez \& Hasbleidy (2014), Osorio (2016) coinciden en que el proceso estocástico o aleatorio se explica como la sucesión de eventos que se desarrolla en el tiempo, en el cual el resultado en cualquier etapa contiene algún elemento que depende del azar. Para el estratega de mercadotecnia resulta trascendente comprender la combinación de estos dos elementos: los modelos de análisis estocástico y los resultados que de su aplicación se derivan, fundamentalmente por los beneficios que, para la toma de decisiones, solución de problemas y diseño de estrategias mercadológicas.

Es importante para la industria conocer en el mercado mexicano ¿cómo la marca interactúa en las decisiones de compra verificando si las generalidades planteadas por Ehrenberg (1970, 1990, 2004), Cannon, Ehrenberg y Goodhardt (1970), Fader y Schmittlein (1993) y Bhattacharya (1997), en relación a las compras repetitivas, (recompra de la misma marca), duplicación de marca (recompra de marcas diferentes), lealtad compartida (lealtad a más de una marca) y doble riesgo (lealtad anclada a las cuotas de mercado), ¿Si éste comportamiento puede ser analizado a través de los modelos estocásticos de elección de compra?,

\section{Marco teórico}

El tema de lealtad del consumidor hacia la marca/producto ha sido analizado durante muchas décadas desde diferentes corrientes; entre ellos destacan Delgado (2004), Colmenares (2007), Moisescu \& Allen (2010), Chrysochou \& Giraud, (2012), Tobón, \& Pérez. (2016). Griffiths (2016).

Estos investigadores consideran que la lealtad se manifiesta como la recompra persistente de una marca a lo largo del tiempo, pero también, toma la forma de un compromiso psicológico que se traduce en una actitud positiva y en una intención de recompra. Para Jensen \& Hansen (2006) la lealtad a la marca está asociada a elevadas cuotas de mercado y altas tasas de rendimiento sobre la inversión. Petroll, Damacena \& Hernani, (2012) Guale, \& Beltrán, 
(2017); coinciden en que la lealtad a la marca también está asociada a la mayor tolerancia de los consumidores a variaciones en la calidad de los productos, a la reducción de costos en la búsqueda de información para la decisión de compra, a la mayor resistencia de los consumidores a promociones de las marcas competidoras y a la reducción de la sensibilidad a cambios de precio. Fischer \& Espejo, (2017) asocian la lealtad a la marca y a la satisfacción del cliente, aunque la satisfacción del cliente no es garantía de lealtad .

Un proceso estocástico sirve para caracterizar una sucesión de variables aleatorias (estocásticas) que evolucionan en función de otra variable, generalmente el tiempo; cada una de las variables aleatorias del proceso tiene su propia función de distribución de probabilidad y entre ellas pueden estar correlacionadas o no (Bourguignon, 1974).

A lo largo de la revisión de la literatura, referente a los modelos estocásticos de elección de compra, no se encontraron investigaciones aplicadas en el contexto mexicano, por lo que la presente investigación busca aceptar o rechazar las generalizaciones propuestas originalmente por Ehrenberg (1970, 1990, 2004), Cannon, Ehrenberg y Goodhardt (1970), Fader y Schmittlein (1993) y Bhattacharya (1997). Y utilizadas en diversos estudios recientes como los realizados por Riquelme (2016), Sánchez \& Hasbleidy (2014), Téllez-León \& Venegas-Martínez (2016), Ortigosa \& Gil (2016), De Souza, Mota, Piñeyro, Fernández \& Fagundes (2016), Muñoz, Muñoz \& Ramírez (2016), En relación a la lealtad de las marcas en compras repetitivas, (recompra de la misma marca), duplicación de marca (recompra de marcas diferentes), lealtad compartida (lealtad a más de una marca) y doble riesgo (lealtad anclada a las cuotas de mercado), utilizando modelos estocásticos para definir el comportamiento de elección de compra

\section{Generalizaciones}

1. Para cualquier par de marcas de consumo frecuente, y en un período de 12 semanas, la proporción de compradores de la marca $X$ que también compra la marca $\mathrm{Y}$, es directamente proporcional a la penetración de la marca Y.

2. Las frecuencias medias de compra de los consumidores duplicantes son las mismas para cualquier par de marcas.

3. En igualdad de circunstancias las pequeñas marcas (menos populares o con menor cuota de mercado) generalmente conservan menos lealtad entre sus clientes que las grandes marcas (más populares o con mayor cuota de mercado).

4. Hay una relación directa entre la penetración y la duplicidad de la marca; es decir, las grandes marcas suelen compartir en promedio más clientes entre si y menos con las pequeñas marcas. 
5. El coeficiente de duplicidad es constante para todas las parejas de marcas.

6. En períodos relativamente largos, como pueden ser doce semanas o más, los valores de los coeficientes de duplicidad son siempre superiores a 1 y en períodos cortos (dos a cuatro semanas) generalmente son menores a 1 . ¿La proporción de compradores de la marca X, que también compra la marca $\mathrm{Y}$, es directamente proporcional a la penetración de la marca Y?

\section{Modelos explicativos}

Los modelos estocásticos (probabilísticos) se dividen en dos grupos: los modelos de incidencia de compra y los modelos de elección de marca. Howard desde 1963 señaló que los modelos de incidencia de compra se utilizan para predecir cuándo se producirá la compra de la marca o cuántas compras se realizarán en un intervalo de tiempo específico. Por su parte, Riquelme (2016) señala que los modelos de elección de marca ayudan al investigador a predecir qué marca (o grupo de marcas) comprarán los consumidores; para estos autores la distinción entre los modelos de elección de marca e incidencia de compra es importante, y para encontrar las diferencias entre estos tipos de modelos se define la siguiente relación:

Sea $B_{i}$ la compra de la marca i, y Pr la compra de cualquier marca de una categoría de producto, la probabilidad de que una persona compre la marca $\mathrm{i}$ en el intervalo de tiempo entre $\mathrm{t}$ y $\mathrm{t}+\mathrm{h}$ es:

$\operatorname{Pr}\left\{B_{i} \in(\mathbf{t}, \mathbf{t}+\mathbf{h})\right\}$

Si esta probabilidad se amplia de acuerdo con la regla de la probabilidad condicional se obtiene:

$\operatorname{Pr}\left\{B_{i} \in(\mathbf{t}, \mathbf{t}+\mathbf{h})\right\}=\operatorname{Pr}\left\{B_{i} \mid \mathbf{P} \in(\mathbf{t}, \mathbf{t}+\mathbf{h})\right\} \operatorname{Pr}\{\mathbf{P} \in(\mathbf{t}, \mathbf{t}+\mathbf{h})\}$

En la ecuación (2), la fracción $\operatorname{Pr}\left\{B_{i} \mid \mathbf{P} \in(\mathbf{t}, \mathbf{t}+\mathbf{h})\right\}$ representa la probabilidad de que una marca sea seleccionada, y la fracción $\operatorname{Pr}\{\mathbf{P} \in(\mathbf{t}, \mathbf{t}+$ h)\} representa la probabilidad de la incidencia de la compra; entonces, los modelos que buscan predecir $\operatorname{Pr}\left\{B_{i} \in(\mathbf{t}, \mathbf{t}+\mathbf{h})\right\}$ son conocidos como modelos de selección de marca y los modelos que buscan predecir $\operatorname{Pr}\{\mathbf{P} \in$ $(\mathbf{t}, \mathbf{t}+\mathbf{h})\}$ son conocidos como modelos de incidencia de compra.

\section{Modelos de incidencia de compra}

Estos modelos se utilizan para predecir el momento en que se producirá la compra, entre la gran variedad de modelos de incidencia de compra destacan tres: el modelo de distribución Binomial Negativa (NBD, por sus siglas en inglés), el modelo Logarithmic Series Distribution (LSD, por sus siglas en inglés) y el modelo Expresiones Aproximadas (EA).

a) Modelo Distribución Binomial Negativa (NBD)

Ehrenberg desde 1964 determinó que el proceso estocástico o aleatorio, entendido como la sucesión de eventos que se desarrolla en el 
tiempo, el resultado en cualquier etapa contiene algún elemento que depende del azar y propone una fórmula que permite estimar tanto la proporción de clientes fieles como predecir la lealtad por repetición de compra. La fórmula en sí es un modelo matemático que implica la distribución binomial negativa, formado con dos dimensiones que considera que las compras de cualquier consumidor sigue una distribución de Poisson a lo largo del tiempo, y que las diversas tasas promedio de compra de todos los diferentes consumidores en el largo plazo siguen una distribución de probabilidad continua $\left(\mathrm{X}^{2}\right)$. El valor principal del modelo matemático radica en aspectos específicos de los patrones de compra que pueden presentar los consumidores; es decir, que, las personas que compran una marca durante un tiempo o período dado, un cierto número de compradores leales comprará de nuevo la misma marca en otro período posterior; cabe aclarar que la proporción de compradores que son leales en ambos períodos se puede predecir a partir de la fórmula matemática que utiliza datos observados relativos a un solo período.

\section{b) Modelo Logarithmic Series Distribution (LSD)}

En el campo de estudio de la conducta de compra del consumidor, Chatfield, Ehrenberg y Goodhardt (1966) profundizaron en el diseño de modelos que permitieran medir la repetición de compra y la sustitución de marca con mayor sencillez para los mercadólogos, y con mayor confiabilidad y validez para los investigadores; fue así que en sustitución de la teoría NBD incorporaron la teoría LSD. En una de sus investigaciones los autores utilizaron el modelo LSD para representar la distribución de los números de las unidades de un producto adquirido por un comprador en un período específico. La aplicación reportó una muy estrecha aproximación al modelo NBD en ciertos rangos de parámetros (más o menos, un valor del exponente $\mathrm{k}$ NBD menos de 0.2); más adelante Ehrenberg, Uncles y Goodhardt (2004) confirmaron la propuesta de incorporación argumentando además que ciertos parámetros del modelo LSD se calculan con rangos muy cercanos a los proporcionados por el modelo NBD, pero principalmente porque el primero resulta ser más simple de operar ya que tiene sólo un parámetro en lugar de dos; otra característica fundamental señalada por los autores acerca del modelo LSD es que sólo afecta a la distribución de los compradores, los nocompradores pueden tratarse por separado. Recientemente se han realizado diversas investigaciones tomando como base este modelo, donde se destaca las realizadas por Castro, Gutiérrez, Araque \& Montero (2016), Grijalba (2017), Velázquez, Farrera \& Cortazar (2017).

\section{Modelo Expresiones Aproximadas (EA)}

Baeza (2010) señala que en un contexto amplio, las expresiones aproximadas son una herramienta complementaria de un modelo riguroso, gracias a que exponen de manera explícita las relaciones entre las variables de 
mayor significación; y en este orden de ideas Ehrenberg et al. (2004), así como Landeta, Cortés \& García, (2016) aseguran que ciertos parámetros del modelo LSD pueden simplificarse aún más en la frecuencia media de compra individual con ayuda del modelo EA.

\section{Modelos de elección de marca}

Lilien, Kotler y Moorthy , (1992) afirman que los modelos estocásticos de elección de marca se distinguen por la influencia que ejerce el comportamiento de elección actual sobre la probabilidad del futuro comportamiento de elección de la marca; para los autores destacan tres modelos de elección de marca: los modelos de orden cero, los modelos de primer orden y los modelos lineales de aprendizaje.

\section{a) Modelos de orden cero}

Los modelos de orden cero suponen que un consumidor, independientemente de las influencias a las que esté expuesto, tiene una probabilidad de compra de marca constante; Para (Lilien et al., 1992). González, Pirovani, Piagentini, Ulín, Miranda-Cruz, Osorio \& Salinas (2016), los modelos de orden cero la probabilidad de compra de la marca en la siguiente ocasión es igual a la probabilidad de compra de la enésima ocasión.

Pinilla (2009) señala que el modelo estocástico de orden cero más simple para describir el comportamiento del consumidor es el Proceso de Bernoulli (1971), mismo que asegura que en un estudio sólo aparecen dos posibles resultados: éxito y fracaso; a la probabilidad de éxito se le representa con $\mathrm{p}$, es decir, $\mathrm{P}(\mathrm{X}=1)=\mathrm{p}$, donde $0 \leq \mathrm{p} \leq$; por su parte, a la probabilidad del fracaso se le representa con $q$, es decir, $\mathrm{P}(\mathrm{X}=0)=\mathrm{q}$; por lo tanto, $\mathrm{p}+\mathrm{q}=1$. Si la recolección de datos se lleva a cabo varias veces y las pruebas son independientes entre sí se conforma un experimento de Bernoulli.

El proceso estocástico $\{\mathbf{X t}, \mathbf{t} \in \mathbf{T}\}, \mathbf{X t} \in \mathbf{R}$

Donde $\mathrm{R}=(0,1)$, y $\mathrm{T}=1,2,3 \ldots$ es un proceso de Bernoulli, si solo si...

$$
\mathrm{P}\left[X_{t}=1 \mid X_{t-1}, X_{t-2}, \ldots, X_{t-p}\right]=p_{i}
$$

En el caso del comportamiento de compra, la fórmula (3) implica que la probabilidad $\boldsymbol{p}_{\boldsymbol{i}}$ es constante en el tiempo e independiente de las decisiones de compra reales del consumidor en el pasado; así, en cualquier decisión de compra (en una categoría de producto en particular) el consumidor tiene la misma probabilidad $\boldsymbol{p}_{\boldsymbol{i}}$ de compra de la marca i. Similar a otros modelos, el modelo de Bernoulli del comportamiento de compra intenta identificar, explicar, o tener en cuenta la heterogeneidad de la población.

Un modelo "conjunto" de orden cero más completo fue propuesto por Ehrenberg (1970); el autor postuló que la probabilidad de la compra conjunta de las marcas i y j en ocasiones sucesivas de compra la probabilidad está dada por 


$$
\mathbf{p}(\mathbf{i}, \mathbf{j})=\mathbf{k m}_{i} \mathbf{m}_{j}
$$

Donde $\mathbf{m}_{\boldsymbol{i}}$ son las participaciones de mercado de las respectivas marcas y $\mathbf{k}$ una constante definida; para el autor resulta fácil demostrar que

$$
\mathbf{p}(\mathbf{i}, \mathbf{i})=\mathbf{m}_{i}-\mathbf{k m}_{i}\left(\mathbf{1}-\mathbf{m}_{i}\right)
$$

Entonces, resumiendo la ecuación (5) anterior obtenemos una ecuación para k:

$$
\mathbf{k}=\frac{1-\sum \mathbf{p}(\mathbf{i}, \mathbf{i})}{1-\sum_{\mathbf{i}} \mathbf{m}_{\mathbf{i}}^{2}}
$$

\section{b) Modelos de primer orden}

Thierauf y Grosse (1972) señalan que un proceso se reconoce como de primer orden cuando tiene un número finito de estados y todos los estados individuales dependen sólo de sus estados anteriores, y que en el caso del comportamiento de compra el modelo Cadenas de Markov es el más significativo. Howard (1971) señala que el caso más simple de un proceso estocástico en que los resultados dependen de otros ocurre cuando el resultado en cada etapa sólo depende del resultado de la etapa anterior y no de cualquiera de los resultados previos, tal proceso se denomina proceso o cadena de Markov (una cadena de eventos, donde cada evento está ligado al precedente). Estas cadenas reciben su nombre por el matemático ruso Andrei Andreevitch Markov (1856-1922) y, como señala el autor, las cadenas tienen memoria, recuerdan el último evento y eso condiciona las posibilidades de los eventos futuros (Thierauf et al., 1972).

Este tipo de proceso presenta una forma de dependencia simple, pero muy útil en muchos modelos entre las variables aleatorias que forman un proceso estocástico; por ejemplo, se utilizan para analizar patrones de compra de marcas, para planear necesidades de personal, para analizar el reemplazo de un equipo, entre otros.

La característica "primer orden" de una cadena de Markov significa que $\boldsymbol{p}_{\boldsymbol{i} \boldsymbol{j}}$ es independiente de los estados individuales en los tiempos $\mathrm{t}-1, \mathrm{t}$ $2, \ldots$; por lo tanto, en este caso el modelo de Markov asume que sólo la última marca elegida afecta a la compra actual; en concreto, hay dos propiedades que caracterizan a un proceso estacionario de primer orden de Markov, sea $\boldsymbol{Y}_{\boldsymbol{t}}$ la marca elegida en la ocasión de compra $\mathbf{t}$ y $\mathbf{N}$ el número de marcas, entonces el modelo de Markov satisface las siguientes condiciones (Thierauf et al., 1972):

$$
\mathbf{p}\left(Y_{t}=\mathbf{k} \mid Y_{t-1}, Y_{t-2} \ldots, Y_{0}\right)=\mathbf{p}\left(Y_{t}=\mathbf{k} \mid Y_{t-1}\right) \quad \text { Un }
$$

período de memoria (7)

$$
\mathbf{p}\left(\boldsymbol{Y}_{\boldsymbol{t}}=\mathbf{k} \mid \boldsymbol{Y}_{\boldsymbol{t}-\mathbf{1}}\right)=\mathbf{p}\left(\boldsymbol{Y}_{\mathbf{1}}=\mathbf{k} \mid \boldsymbol{Y}_{\mathbf{0}}\right) \quad \text { Estacionario para todos }
$$
los $\mathrm{t}, \mathrm{k}$ (7)

\section{c) Modelos lineales de aprendizaje (LA)}

Para Kuehn (1962), estos modelos estocásticos de elección de marca consideran la conducta adaptativa de los consumidores; la idea básica que le 
llevó al desarrollo de un modelo de este tipo es que los consumidores se ven afectados por la retroalimentación de las elecciones de marca del pasado; por lo tanto, se supone que el acto de compra de una marca en particular afecta la probabilidad de que esta marca se seleccionará la próxima vez. Para Zhang, Siebers \& Aickelin (2016) los modelos lineales de aprendizaje ofrecen un conjunto de hipótesis sobre la forma en que un evento de compra retroalimenta las probabilidades posteriores a la compra; la suposición más importante es que la probabilidad de la compra posterior es siempre una función lineal de la probabilidad de la compra anterior, esta función lineal tiene la siguiente forma:

$$
\text { pt }=\alpha+\beta p_{t-1}
$$

Para todos los valores de $\mathbf{p t}$ y todo $\mathbf{t}, \mathrm{y} \boldsymbol{\alpha}, \boldsymbol{\beta} \in[\mathbf{0}, \mathbf{1}]$.

La parte derecha de la ecuación $\left(\boldsymbol{\alpha}+\boldsymbol{\beta} \boldsymbol{p}_{\boldsymbol{t}-\mathbf{1}}\right)$ se llama operador de retroalimentación.

Supuestos adicionales contenidos en modelos lineales de aprendizaje son:

El modelo asume cuasi-estacionariedad en el sentido de que los parámetros de los operadores de cambio no se alteran en períodos cortos de tiempo.

El modelo asume que todos los individuos/hogares tienen una conducta adaptativa que puede ser descrita por los operadores de retroalimentación (compras anteriores) con los mismos parámetros; por lo tanto, el parámetro en el modelo lineal de aprendizaje se supone es el mismo para todos los individuos/hogares.

\section{Método}

Con el objetivo de medir a través de modelos estocásticos la elección de compra recurrente en hogares de la Ciudad de México y confirmar o rechazar seis generalizaciones relacionadas con las conductas de compra repetida (recompra de la misma marca), duplicación de marca (recompra de marcas diferentes), lealtad compartida (lealtad a más de una marca) y doble riesgo (lealtad anclada a las cuotas de mercado); se tomó una muestra de hogares mexicanos radicados en la ciudad de México para determinar y medir sus comportamientos de compra de productos de consumo recurrente. Se realizó un estudio cuantitativo, de naturaleza longitudinal/transversal a través de panel de hogares. el muestreo fue por juicio debido a que solo se utilizó 60 hogares que pertenecían a un salón de clase de la Licenciatura en Administración de la Facultad de Contaduría y Administración de la UNAM; los hogares de los participantes pertenecían a la zona sur de la Ciudad de México (ver Tabla 1); los alumnos aceptaron colaborar semanalmente con la entrega del ticket de compra de productos alimenticios no perecederos y de higiene; además de la presentación del ticket de compra, cada participante vaciaba la información en un formato Excel separado por categorías: de 
producto, precio, tipo de artículos y lugar de compra. El estudio fue llevado a cabo durante 12 semanas en los meses de septiembre a noviembre del 2015;

Al finalizar la $12^{\circ}$ semana, se recopilo toda la información de cada uno de los 60 hogares participantes se obtuvo una hoja Excel general y con ella se procedió al análisis de la información.

Tabla 1. Residentes en la zona sur del área metropolitana de la Ciudad de México

\begin{tabular}{|c|c|c|c|c|}
\hline Hogares & $\mathrm{AB}$ & $\mathrm{C}+$ & $\mathrm{C}$ & $\mathrm{C}-$ \\
\hline Participantes & 4 & 16 & 13 & 27 \\
\hline Proporción & $6.3 \%$ & $26.3 \%$ & $20.9 \%$ & $45.0 \%$ \\
\hline AMAI $^{1}$ Nacional & $7.6 \%$ & $13.7 \%$ & $17.0 \%$ & $17.1 \%$ \\
\hline Hogares DF & 186,430 & 336,065 & 417,015 & 419,468 \\
\hline
\end{tabular}

Para el análisis general, se seleccionaron cinco categorías consideradas representativas de los hábitos alimenticios y de higiene de los hogares del Distrito Federal tomadas de las categorías alimentos, higiene, belleza y uso doméstico pertenecientes al canasto de productos Nielsen (Canasto Nielsen, 2014): cereal, yogurt, papel higiénico, shampoo para cabello y atún enlatado; los datos de la categoría cereal fueron tabulados por marca de fabricante; para el resto de las categorías los datos se tabularon por marca de producto, resultados que se muestran en la tabla 2 .

\section{Hallazgos}

Para el análisis específico por categoría de producto se seleccionó únicamente el cereal, realizando los parámetros estocásticos. La tabla 3 muestra los parámetros NDB, LSD, EA y Markov observados en un período de doce semanas en las marcas de la categoría cereales, y a partir de éstos la tabla 4 presenta los valores teóricos para penetración, frecuencia media de compra y proporción de repetidores correspondientes a las marcas de la categoría cereales.

${ }^{1}$ Asociación Mexicana de Agencias de Investigación de Mercado y Opinión Pública (AMAI, 2015). 
Tabla 2. Parámetros descriptivos de una semana típica dentro de un período de 12 semanas

\begin{tabular}{|r|r|c|c|c|c|c|}
\hline \multicolumn{2}{|c|}{ Parámetros descriptivos } & Cereal & Yogurt & Papel Higiénico & Shampoo & Atún \\
\hline 1 & \# de marcas monitoreadas & 7 & 14 & 20 & 29 & 5 \\
\hline 2 & \# de familias panelistas & 60 & 60 & 60 & 60 & 60 \\
\hline 3 & \# de visitas de compra promedio & 18.3 & 28.1 & 10.9 & 4.7 & 24.2 \\
\hline 4 & \# de familias compradoras promedio & 9.1 & 10.9 & 4.6 & 3.1 & 10.8 \\
\hline 5 & Penetración promedio & $15.2 \%$ & $18.1 \%$ & $7.7 \%$ & $5.1 \%$ & $18.0 \%$ \\
\hline 6 & Participación promedio & $14.3 \%$ & $7.1 \%$ & $5.0 \%$ & $3.4 \%$ & $20.0 \%$ \\
\hline 7 & Frecuencia media de compra & 2.0 & 2.6 & 2.4 & 1.5 & 2.2 \\
\hline 8 & Hogares repetidores promedio & 4.6 & 6.9 & 6.7 & 6.7 & 6.6 \\
\hline 9 & \% promedio de hogares repetidores & $7.6 \%$ & $11.4 \%$ & $11.2 \%$ & $11.2 \%$ & $11.0 \%$ \\
\hline 10 & Duplicidad de marca promedio & $15.6 \%$ & $19.5 \%$ & $7.6 \%$ & $7.2 \%$ & $19.1 \%$ \\
\hline 11 & Coeficiente de Duplicidad promedio & 1.03 & 1.08 & 0.99 & 0.86 & 0.66 \\
\hline 12 & Duplicidad teórica (pronóstico) promedio & $14.6 \%$ & $7.7 \%$ & $5.0 \%$ & $3.0 \%$ & $13.2 \%$ \\
\hline 13 & Duplicidad promedio entre pares de marcas & 1.41 & 1.04 & 0.53 & 1.48 & 0.87 \\
\hline 14 & \% de compradores únicos & $46.7 \%$ & $18.3 \%$ & $41.7 \%$ & $40.0 \%$ & $36.7 \%$ \\
\hline 15 & \% de compradores leales a 2 marcas & $25.0 \%$ & $31.7 \%$ & $26.7 \%$ & $16.7 \%$ & $26.7 \%$ \\
\hline 16 & \% de compradores leales a 3 marcas & $3.3 \%$ & $15.0 \%$ & $11.7 \%$ & $10.0 \%$ & $0.0 \%$ \\
\hline 17 & Cuota de cartera (\% del valor del ticket) & $2.85 \%$ & $4.75 \%$ & $4.23 \%$ & $2.92 \%$ & $2.84 \%$ \\
\hline 18 & & & & $1.7 \%$ & $5.0 \%$ & $0.0 \%$ \\
\hline 19 & $23.3 \%$ & $6.7 \%$ & $3.3 \%$ & $5.0 \%$ & $0.0 \%$ \\
\hline 20 & & & & $23.3 \%$ & $36.7 \%$ \\
\hline
\end{tabular}

Tabla 3. Parámetros NDB, LSD, EA y Markov para la categoría cereal para un período de 12 semanas

\begin{tabular}{|c|c|c|c|c|c|c|c|c|c|}
\cline { 2 - 10 } \multicolumn{1}{c|}{} & \multicolumn{4}{|c|}{ Datos observados } & \multicolumn{5}{c|}{ Estadísticos derivados } \\
\hline Marcas & $b$ & $w$ & $p_{0}=1-b$ & $m=b w$ & $c=-m / \ln \left(\mathrm{p}_{0}\right)$ & $a^{*}$ & $k=m / a$ & $q^{* *}$ & $q 1$ \\
\hline Nestlé & $31.7 \%$ & 2.21 & 0.68 & 0.700 & 1.84 & 2.047 & 0.34 & 0.759 & 0.672 \\
\hline Kelloggs & $56.7 \%$ & 2.09 & 0.43 & 1.183 & 1.42 & 0.928 & 1.28 & 0.735 & 0.481 \\
\hline Post & $1.7 \%$ & 2.00 & 0.98 & 0.033 & 1.98 & 2.462 & 0.01 & 0.715 & 0.711 \\
\hline Granvita & $3.3 \%$ & 1.50 & 0.97 & 0.050 & 1.47 & 1.077 & 0.05 & 0.534 & 0.519 \\
\hline Aurrera & $6.7 \%$ & 1.50 & 0.93 & 0.100 & 1.45 & 1.014 & 0.10 & 0.534 & 0.503 \\
\hline
\end{tabular}

Tabla 4. Valores teóricos (pronósticos). Categoría cereal

\begin{tabular}{|c|c|c|c|c|c|c|c|c|c|c|}
\cline { 2 - 11 } \multicolumn{1}{c|}{} & \multicolumn{4}{|c|}{ Penetración una Semana típica } & \multicolumn{3}{c|}{ Frecuencia media de compra c/4 sem } & \multicolumn{3}{c|}{ Proporción de repetidores c/12 semanas } \\
\hline Marcas & $\boldsymbol{b}_{T}{ }^{1}$ (NBD) & $b_{T}($ LSD $)$ & $b_{T}$ (Aprox.) & Markov & $w^{2}$ (NBD) & $w$ (LSD) & $w$ (Aprox.) & $b_{R}{ }^{3}$ (NBD) & $b_{R}$ (LSD) & $b_{R}$ (Aprox.) \\
\hline Nestlé & $5.2 \%$ & $5.2 \%$ & $5.0 \%$ & $2.8 \%$ & 1.43 & 1.46 & 1.49 & $65.3 \%$ & $60.3 \%$ & $59.3 \%$ \\
Kelloggs & $9.0 \%$ & $8.8 \%$ & $8.6 \%$ & $4.8 \%$ & 1.36 & 1.41 & 1.44 & $70.0 \%$ & $58.5 \%$ & $57.2 \%$ \\
Post & $0.3 \%$ & $0.3 \%$ & $0.2 \%$ & $0.2 \%$ & 1.37 & 1.38 & 1.41 & $57.3 \%$ & $57.0 \%$ & $55.6 \%$ \\
Granvita & $0.4 \%$ & $0.4 \%$ & $0.4 \%$ & $0.3 \%$ & 1.18 & 1.18 & 1.20 & $44.4 \%$ & $44.0 \%$ & $40.8 \%$ \\
Aurrera & $0.8 \%$ & $0.8 \%$ & $0.8 \%$ & $0.3 \%$ & 1.18 & 1.18 & 1.20 & $44.9 \%$ & $44.0 \%$ & $40.8 \%$ \\
\hline
\end{tabular}

\section{Notaciones:}

$\boldsymbol{b}_{\boldsymbol{T}} \quad \begin{gathered}\text { Penetración teórica } \\ \text { Compra }\end{gathered}$
Frecuencia Media de


$\boldsymbol{b}_{\boldsymbol{R}} \quad$ Proporción de repetidores

MKV Modelo cadenas de Markov

NBD Modelo Distribución Binomial Negativa

LSD Modelo Distribución Logarítmica

EA Modelo de Expresiones Aproximadas

Los resultados presentados en las tablas 3 y 4 sugieren las siguientes interpretaciones:

1. Dado que el $31.7 \%$ de la muestra compró la marca Nestlé, en promedio 2.21 veces en el período de doce semanas (Tabla 3), la estimación NBD es que alrededor del $5.2 \%$ de la muestra debe comprar la marca Nestlé en una semana típica (tabla 4);

2. Asimismo, la estimación NBD muestra que la frecuencia media con la cual los compradores de la marca la adquirirán cada cuatro semanas es de alrededor de 1.43 veces (Tabla 4); y

3. La estimación NBD también señala que la proporción de compradores que repetirán la compra en un mismo período de doce semanas es alrededor del $65.3 \%$ (Tabla 4), proporción que también puede entenderse como índice de lealtad a la marca.

\section{a) Duplicación de marca (recompra de marcas diferentes).}

En relación con la primera generalización: "Para cualquier par de marcas de consumo frecuente, y en un periodo de 12 semanas, la proporción de compradores de la marca $X$ que también compra la marca $Y$, es directamente proporcional a la penetración de la marca $Y$ ", los resultados obtenidos muestran que las duplicidades pareadas, observadas entre las marcas de la categoría cereales, se presentan en la Tabla 5. Después de analizar las cifras, se observa que representan la proporción de compradores de la marcafila que también compran la marca-columna; por ejemplo, en el recuadro interno se aprecia que la proporción de compradores de Kellogg's, que también compran Nestlé, es el $35.3 \%$; por su parte, la proporción de compradores de Nestlé, que también compra Kellogg's, es el 63.2\%; analizados en forma individual, ambos porcentajes de duplicidad lucen directamente proporcionales a la penetración de las marcas mostradas en las celdas en diagonal (fondo gris); estos resultados permiten aceptar la primera generalización. 
Tabla 5. Duplicidades pareadas. Categoría cereal

\begin{tabular}{|c|c|c|c|c|c|c|c|}
\cline { 2 - 8 } \multicolumn{1}{c|}{} & Kelloggs & Nestlé & Maizoro & Granvita & Quaker & Aurrera & Post \\
\hline Kelloggs & $56.7 \%$ & $35.3 \%$ & $0.0 \%$ & $2.9 \%$ & $0.0 \%$ & $5.9 \%$ & $2.9 \%$ \\
\hline Nestlé & $63.2 \%$ & $31.7 \%$ & $0.0 \%$ & $10.5 \%$ & $5.3 \%$ & $5.3 \%$ & $0.0 \%$ \\
\hline Maizoro & $0.0 \%$ & $0.0 \%$ & $1.7 \%$ & $0.0 \%$ & $100.0 \%$ & $0.0 \%$ & $0.0 \%$ \\
\hline Granvita & $50.0 \%$ & $100.0 \%$ & $0.0 \%$ & $3.33 \%$ & $0.0 \%$ & $0.0 \%$ & $0.0 \%$ \\
\hline Quaker & $0.0 \%$ & $33.3 \%$ & $33.3 \%$ & $0.0 \%$ & $5.00 \%$ & $0.0 \%$ & $0.0 \%$ \\
\hline Aurrera & $50.0 \%$ & $25.0 \%$ & $33.3 \%$ & $0.0 \%$ & $0.0 \%$ & $6.67 \%$ & $0.0 \%$ \\
\hline Post & $100.0 \%$ & $0.0 \%$ & $0.0 \%$ & $0.0 \%$ & $0.0 \%$ & $0.0 \%$ & $1.67 \%$ \\
\hline
\end{tabular}

\section{b) Frecuencia media de compra pareada}

En referencia a la segunda generalización: "Las frecuencias medias de compra de los consumidores duplicantes son las mismas para cualquier par de marcas", la tabla 6 muestra, para un período de 12 semanas, los valores de las frecuencias promedio de compra para todos los pares posibles entre las marcas de cereal; su interpretación es la siguiente, por ejemplo, la cifra 1.7 (en el cruce de la columna Nestlé con el renglón Kellogg's) indica que las personas que compran estas dos marcas compran la marca Nestlé 1.7 veces en un período de doce semanas; por su parte la cifra 2.4 (en el cruce de la columna Kellogg's con el renglón Nestlé) indica que las personas que compran las mismas dos marcas, compran la marca Kellogg's 2.4 veces en el mismo período de doce semanas; la frecuencia media de compra para todos los compradores de cada marca se muestra en las celdas en diagonal (fondo gris), con lo que se acepta así la generalización planteada.

Tabla 6 Frecuencia media de compra pareada. Categoría cereal

\begin{tabular}{|c|c|c|c|c|c|c|c|c|c|}
\hline & Kelloggs & Nestlé & Aurrera & Quaker & Granvita & Maizoro & Post & Promedios & Participación \\
\hline Kelloggs & 2.09 & 1.7 & 1.0 & 0.0 & 2.0 & 0.0 & 2.0 & 1.1 & $53.1 \%$ \\
\hline Nestlé & 2.4 & 2.21 & 1.0 & 1.0 & 1.5 & 0.0 & 0.0 & 1.0 & $29.7 \%$ \\
\hline Aurrera & 2.0 & 6.0 & 1.50 & 0.0 & 0.0 & 0.0 & 0.0 & 1.3 & $6.3 \%$ \\
\hline Quaker & 0.0 & 1.0 & 0.0 & 1.00 & 0.0 & 1.0 & 0.0 & 0.3 & $4.7 \%$ \\
\hline Granvita & 2.0 & 1.0 & 0.0 & 0.0 & 1.50 & 0.0 & 0.0 & 0.5 & $3.1 \%$ \\
\hline Maizoro & 0.0 & 0.0 & 0.0 & 1.0 & 0.0 & 1.00 & 0.0 & 0.2 & $1.6 \%$ \\
\hline Post & 3.0 & 0.0 & 0.0 & 0.0 & 0.0 & 0.0 & 2.00 & 0.5 & $1.6 \%$ \\
\hline & & & & & & & Desv Std & 0.4 & \\
\hline
\end{tabular}

Para la tercera generalización que afirma: "En igualdad de circunstancias las pequeñas marcas (menos populares o con menor cuota de mercado) conservan menos lealtad entre sus clientes que las grandes marcas (más populares o con mayor cuota de mercado) entre los suyos", a pesar de que las cuotas de mercado observadas (columna participación) son considerablemente diferentes entre las marcas, las frecuencias medias de compra (columna promedios) presentan pequeñas variaciones. Esto sugiere que la tasa promedio de compra con la que cualquier marca es comprada conjuntamente con otra marca (por ejemplo A/B) por los consumidores duplicantes difiere tan sólo en .4 (celda azul) de otra compra duplicada (por ejemplo, A/C, C/D o B/D); en los límites bastante estrechos de variación mostrados en las frecuencias de compra de la tabla 6 se aprecia, sin embargo, 
una tendencia sistemática que, aunque pequeña e irregular, es decreciente (hacia la parte baja de la columna promedios), la tendencia es esencialmente una función de la participación de mercado o del nivel de penetración y específicamente se refiere a la ley Double Jeopardy (doble riesgo), aceptándose así la tercera generalización.

\section{c) Coeficientes de duplicidad.}

Para la cuarta hipótesis planteada en el estudio: "Existe, en general, una relación directa entre la penetración y la duplicidad de la marca; es decir, las grandes marcas suelen compartir en promedio más clientes entre si y menos con las pequeñas marcas", en la Tabla 7 se presentan los coeficientes observados y teóricos de duplicidad de marcas; por ejemplo, para el caso de la marca de cereal Kellogg's la duplicidad observada es $43.9 \%$ y la duplicidad teórica es del 41.1\%; se estima entonces una disminución de 2.8 puntos porcentuales; mientras el coeficiente de duplicidad teórica (41.1\%) significa que se pronostica para las próximas doce semanas que el $41.1 \%$ de los compradores de cualquier otra marca de cereal compren también la marca Kellogg's. Por su parte, la pérdida de puntos porcentuales sugiere que en las próximas doce semanas las estrategias de mercadotecnia de la marca perderán efectividad para atraer a los compradores de otras marcas o que las estrategias de las marcas competidoras están siendo más efectivas en la retención de sus consumidores; también se puede apreciar, recorriendo la vista hacia abajo, que hay diferencias considerables en las duplicaciones observadas; por ejemplo, los compradores duplicantes de las marcas en la parte baja de la tabla reportan duplicidades menores al $10 \%$ mientras que las duplicidades de los compradores de las marcas en la parte superior de la tabla están por arriba del 20\%. Estas diferencias en las duplicidades entre las marcas de una categoría sugieren partición del mercado; es decir, la existencia de segmentos de mercado o clusters de marcas donde la competencia entre ellas es más intensa. Asimismo, se muestra la correlación duplicidad-penetración que permitirá aceptar o rechazar la hipótesis planteada.

Tabla 7. Coeficientes de duplicidad de marcas, observada y teórica. Categoría cereal

\begin{tabular}{|c|c|c|c|}
\hline \multicolumn{4}{|c|}{ Cereal } \\
\hline Marcas & $\begin{array}{c}\text { Duplicidad } \\
\text { observada }\end{array}$ & $\begin{array}{c}\text { Duplicidad } \\
\text { Teórica }\end{array}$ & Diferencia \\
\hline Kelloggs & $43.9 \%$ & $41.1 \%$ & $-2.8 \%$ \\
\hline Nestlé & $32.3 \%$ & $30.3 \%$ & $-2.0 \%$ \\
\hline Quaker & $17.5 \%$ & $16.4 \%$ & $-1.1 \%$ \\
\hline Maizoro & $11.1 \%$ & $10.4 \%$ & $-0.7 \%$ \\
\hline Granvita & $2.2 \%$ & $2.1 \%$ & $-0.1 \%$ \\
\hline Aurrera & $1.9 \%$ & $1.7 \%$ & $-0.2 \%$ \\
\hline Post & $0.5 \%$ & $0.5 \%$ & $0.0 \%$ \\
\hline
\end{tabular}




\section{d) Correlación duplicidad-penetración}

Cada punto mostrado en la gráfica 1 es una marca de cereal participante en el estudio; se muestra para cada marca la relación que existe entre su duplicidad y su penetración. De igual forma, se puede observar para cada una de las marcas que los niveles promedio de duplicación de compra declinan de derecha a izquierda (eje de las "x") conforme la penetración declina también de arriba hacia abajo (eje de las "y"); éste es el resultado esperado de la ley de la duplicación de la compra de marcas, en otras palabras, el comportamiento mostrado por las marcas en el gráfico

El valor 1.0718 es el coeficiente de duplicación en la compra de marcas (o coeficiente de intercambio) para la categoría. Este valor expresa la relación esperada entre el tamaño de una marca A y la proporción media de los usuarios de otras marcas que se esperaría compraran también la marca A en un período determinado, además, sirve para calcular la duplicación esperada para cada una de las marcas.

$\mathrm{R}^{2}$ se refiere al coeficiente de determinación, y el valor .8868 expresa la proporción de varianza conjunta entre las variables: duplicidad y penetración, se considera que este valor muestra una relación alta entre las variables; por lo tanto, se acepta la cuarta generalización planteada en el estudio.

Grafica 1. Relación entre duplicidad de marca y penetración de marca. Categoría cereal

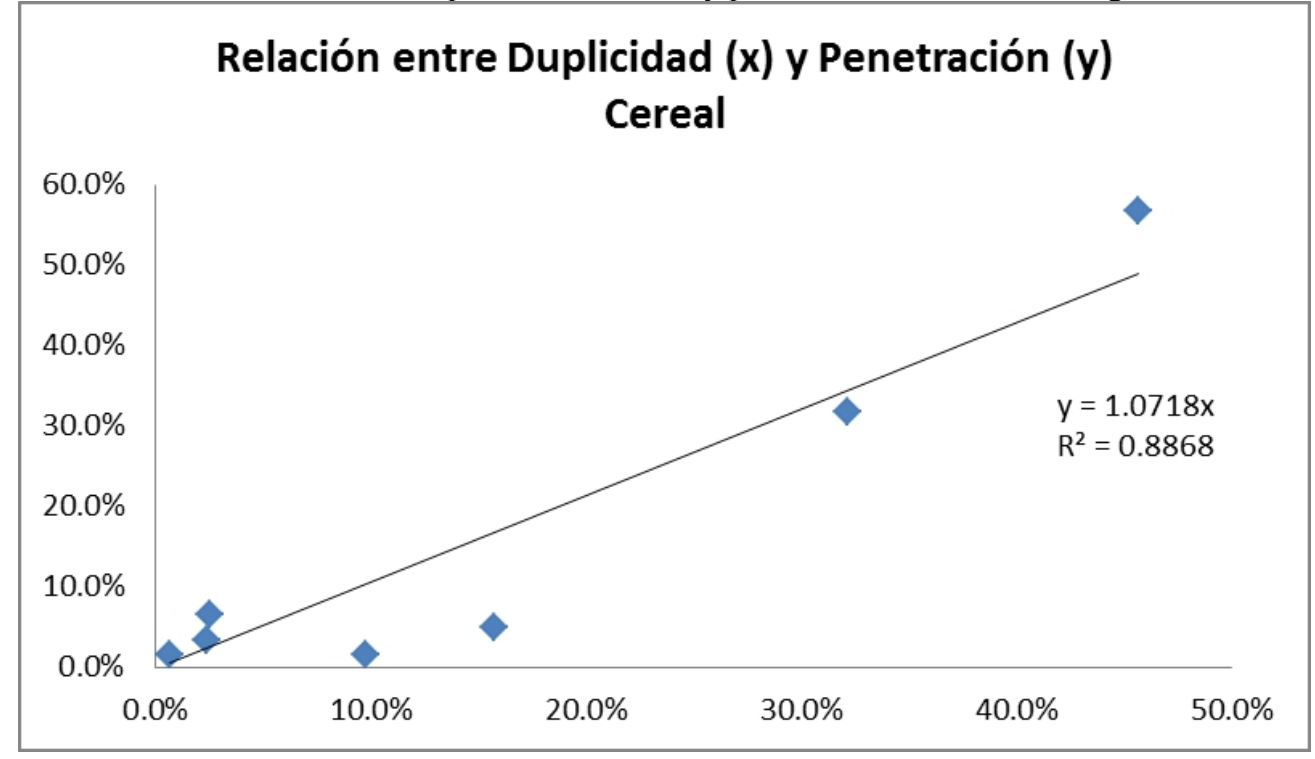

e) Coeficientes de duplicidad entre marcas

La quinta generalización "El coeficiente de duplicidad es aproximadamente constante para todas las parejas de marcas" se ve reflejada en los resultados de la Tabla 8, donde se analizan los coeficientes de duplicidad pareados entre marcas. Para los valores mostrados derivan las 
siguientes interpretaciones: un coeficiente igual a 1 significa que la compra de las marcas A y B no está correlacionada; un coeficiente con valor mayor a 1 significa que los compradores de A son más propensos que el resto de la población para comprar la marca $\mathrm{B}$; y un coeficiente con valor menor a 1 significa que la compra de una marca inhibe la compra de la otra marca. En la tabla 7 se puede apreciar que los coeficientes se comportan relativamente constantes, lo que permite aceptar esta hipótesis; asimismo, para aceptar o rechazar la sexta hipótesis se puede ver que estos valores, para 12 semanas, son mayores a 1, lo que también permite aceptar esta generalización: "En períodos relativamente largos, como pueden ser doce semanas o más, el valor de los coeficientes de duplicidad son siempre superiores a 1 y en periodos cortos (dos a cuatro semanas) generalmente son menores a 1", en las celdas en diagonal (fondo gris) se muestra la penetración de cada una de las marcas.

Tabla 8. Coeficientes pareados de duplicidad. Categoría cereal

\begin{tabular}{|c|c|c|c|c|c|c|c|c|}
\cline { 2 - 9 } \multicolumn{1}{c|}{} & Kelloggs & Nestlé & Maizoro & Granvita & Quaker & Aurrera & Post & Promedio \\
\hline Kelloggs & $56.7 \%$ & 1.1 & 0.0 & 0.9 & 0.0 & 0.9 & 1.8 & 0.77 \\
\hline Nestlé & 1.1 & $31.7 \%$ & 0.0 & 3.2 & 1.1 & 0.8 & 0.0 & 1.02 \\
\hline Maizoro & 0.0 & 0.0 & $1.7 \%$ & 0.0 & 20.0 & 0.0 & 0.0 & 3.33 \\
\hline Granvita & 0.9 & 3.2 & 0.0 & $3.33 \%$ & 0.0 & 0.0 & 0.0 & 0.67 \\
\hline Quaker & 0.0 & 1.1 & 20.0 & 0.0 & $5.00 \%$ & 0.0 & 0.0 & 3.51 \\
\hline Aurrera & 0.9 & 0.8 & 0.0 & 0.0 & 0.0 & $6.67 \%$ & 0.0 & 0.28 \\
\hline Post & 1.8 & 0.0 & 0.0 & 0.0 & 0.0 & 0.0 & $1.67 \%$ & 0.29 \\
\hline Promedio & 0.77 & 1.02 & 3.33 & 0.67 & 3.51 & 0.28 & 0.29 & 1.41 \\
\hline
\end{tabular}

\section{Conclusión}

A través de la medición del consumo de hogares para conocer su tendencia sistemática de compra y sus comportamientos de lealtad en marcas de consumo recurrente, el estudio permitió con la ayuda modelos de análisis estocástico aceptar las seis generalizaciones relacionadas con las conductas de compra repetida (recompra de la misma marca), duplicación de marca (recompra de marcas diferentes), lealtad compartida (lealtad a más de una marca) y doble riesgo (lealtad anclada a las cuotas de mercado) propuestas por Ehrenberg (1970, 1990, 2004), Cannon, Ehrenberg y Goodhardt (1970), Fader y Schmittlein (1993) y Bhattacharya (1997); en donde se destaca la utilización de los modelos estocásticos para analizar el comportamiento de compra repetido y la lealtad a la marca

Los resultados obtenidos permiten de manera científica mostrar a los mercadologos el comportamiento de compra de hogares mexicanos, Aunque los resultados obtenidos no se pueden generalizar a todos los hogares de la Ciudad de México, sí presenta resultados similares a los obtenidos en estudios similares en el extranjero.

Los resultados de esta investigación también reportan que el coeficiente de duplicidad de marcas es un indicador de gran ayuda para el mercadólogo al permitirle identificar con precisión las características y 
preferencias comparativas de los segmentos del mercado que satisfacen sus necesidades y expectativas con las diversas marcas disponibles en una misma categoría de producto, planteando consecuentemente opciones para resolver dilemas de posicionamiento.

\section{Consideraciones finales}

Finalmente, es conveniente señalar que para fines de generalización el mercadólogo debe ser precavido, puesto que los resultados reportados por esta investigación sólo cubren un segmento muy definido del mercado mexicano; sin embargo, consideramos que pueden ser aplicables en otros países con mercados similares, donde la competencia entre las marcas de las categorías alimentos, higiene, belleza y uso doméstico también es importante.

Finalmente, un pendiente adicional es analizar los datos recolectados con ayuda del modelo estocástico de comportamiento de compra "Dirichlet", el cual describe cuándo se realiza una compra de la categoría de producto y qué marca es seleccionada, contrasta con los modelos NBD y LSD en que éstos últimos parten de "cuándo" una marca es comprada.

\section{References:}

1. AMAI (7 de 12 de 2015). Asociación Mexicana de Agencias de Investigación de Mercado y Opinión Pública. Obtenido de AMAI: http://nse.amai.org/nseamai2/

2. Baeza, A. (2010). Química analítica. México: UNAM.

3. Ballester, M. E. D. (2004). Estado actual de la investigación sobre la lealtad a la marca: una revisión teórica. Dirección y Organización (30).

4. Bernoulli, J. (1971). The Art of Conjecturing. Basilea: The Johns Hopkins University Press.

5. Bhattacharya, C. (1997). Is Your Brand's Loyalty Too Much Too Little or Just Right? Explaining Deviations in Loyalty From the Dirichlet Norm. International Journal of Research in Marketing, 14 (5): 42135 .

6. Bourguignon, F. (1974). A Particular Class of Countinuous-Time Stochastic Growth Models. Journal of Economic Theory, 141-58.

7. Cannon, T. E. (1970). Regularities in sole buying. British Journal of Marketing, 4 (2): 80-6.

8. Castro-González, P., Gutiérrez-Villar, B., Araque-Padilla, R. Á., \& Montero-Simó, M. J. (2016). Export intensity and strengths perceived in the marketing strategy: A perspective based on Spanish export companies' experience. Intangible Capital, 12(5): 1484-1504.

9. Cebollada, J. (1996). Comportamiento del consumidor competencia en los mercados y estrategia comercial de las empresas. Aplicaciones empíricas. Barcelona: Universidad Autónoma de Barcelona. 
10. Chatfield, C. E. (1966). Progress on a simplified model of stationary purchasing behavior. Journal of The Royal Statistical Society, Series A, (129): 317-67.

11. Chrysochou, P., Krystallis, A., \& Giraud, G. (2012). Quality assurance labels as drivers of customer loyalty in the case of traditional food products. Food quality and preference, 25(2): 156-162.

12. Colmenares, O. S. (2007). Aproximación teórica de la lealtad de marca: enfoques y valoraciones. Cuadernos de Gestión, 7 (2): 69-81.

13. De Souza Silva, A., Mota, T. A., Piñeyro, N. G., Fernández, M. G., \& Fagundes F. (2016). Spatial Distribution of Vatiga spp.(Hemiptera: Tingidae) on Cassava Crop. Acta Biológica Colombiana, 21(1): 195200.

14. Delgado, M. (2004). Estado actual de la investigación sobre la lealtad a la marca: una revisión teórica. Revista de dirección, organización y administración de empresas Núm.30 Universidad de Murcia, 16-24.

15. Dekimpe, M. G., Steenkamp, J. B. E., Mellens, M., \& Abeele, P. V. (1997). Decline and variability in bra Dekimpe, Steenkamp, Mellens \& Abeele 1997nd loyalty. international Journal of Research in Marketing, 14(5), 405-420.

16. Ehrenberg, A. (1964). Estimating the Proportion of Loyal Buyers. Journal of Marketing Research (1 Feb.): 56-59.

17. Statistics, VIII, 26-41.

18.

(1970). A Model of Multi-Brand Buying. Journal of Marketing Research, VII, 77-84.

19.

(1990). Double Jeopardy Revisited. Journal of Marketing. 54, 82-91.

20.

(2004). Understanding brand performance measures: using Dirichlet benchmarks. Journal of Business Research 57, 130725.

21. Fader, P. S. (1993). Excess Behavioral Loyalty for High-Share Brands: Deviation from the Dirichlet Model for Repeat Purchasing: Deviations from the Dirichlet Model for Repeat Purchase. Journal of Marketing Research, 30 (4): 478-93.

22. Fischer, L. Espejo, J. (2017). Mercadotecnia. México: McGraw-Hill.

23. González-González, G., Pirovani, M. E., Piagentini, A. M., UlínMontejo, F., Miranda-Cruz, E., Osorio-Osorio, R., ... \& SalinasHernández, R. M. (2016). Cinética de cambios sensoriales y vida de anaquel de carambola mínimamente procesada. Revista fitotecnia mexicana, 39(4): 393-402.

24. Griffiths Acha, S. M. (2016). La congruencia entre la personalidad de marca y el autoconcepto, real y deseado, del consumidor, y su impacto 
en la lealtad de marca de las mujeres limeñas, entre 20 y 35 años, de NSE B, dentro del mercado de cosméticos

25. Grijalba Aponte, F. J. (2017). ¿ Cuál es el efecto de la tasa de cambio en la ventas de vehículos desde el año 2000 al 2015? (Bachelor's thesis, Universidad de La Sabana).

26. Guale, R. J. A., \& Beltrán, F. V. (2017). La interacción personal y su efecto en la decisión de compra. Retos, (13), 75-89.

27. Howard, R. (1963). Stochastic Process Models of Consumer Behavior. Journal of Advertising Research, 3 (3, September): 35 - 42.

28. (1965). Dynamic Inference. Journal of the Operations Research Society of America, 13,(2): 712-733.

29. (1971). Dynamic Probabilistic Systems, volume 1: Markov Chains. Appendix B. New York: John Wiley and Sons.

30. Jensen, J. H. (2006). An empirical examination of brand loyalty. Journal of Product \& Brand Management, 15 (7): 442-9.

31. Kuehn, A. (1962). Consumer Brand- Choice - A Learning Process? Journal of Advertising Research, II, 10-17.

32. Landeta, J. M. I., Cortés, C. B. Y., \& García, O. G. (2016). Variabilidad de la demanda del tiempo de entrega, existencias de seguridad y costo del inventario. Contaduría y Administración, 61(3): 499-513.

33. Lilien, G. K. (1992). Marketing Models. New York: Prentice Hall International.

34. Mordecki, E. (2010). Modelos estocásticos en finanzas. Pontificia Universidad Católica del Perú. Departamento de Ciencias. Sección Matemáticas.

35. Moisescu, O. I., \& Allen, B. (2010). The Relationship Between The Dimensions Of Brand Loyalty. An Empirical Investigation Among Romanian Urban Consumers. Management \& Marketing, 5(4): 83.

36. Muñoz, D. F., Muñoz, D. G., \& Ramírez-López, A. (2016). El Problema del Voceador con Incertidumbre Paramétrica. Información tecnológica, 27(4): 183-192.

37. Nielsen

(2014), http://www.nielsen.com/content/dam/corporate/mx/reports/2014/

38. Canastos\%20Nielsen\%20Febrero\%202014.pdf consultada 6 de febrero de 2016

39. Orta, Ó. M. (2001). Comportamiento de compra en el punto de venta, tipología de compradores y leccion de marca (*). Estudios sobre consumo, (58), 9-22.

40. Ortigosa Hernández, M., \& Gil Lafuente, A. M. (2016). Metodología para elaborar leyes de posibilidad de retirada del cliente: una aplicación al sector del vestido//A Methodology to Elaborate Laws of 
Possibilities in the Retreat of a Client: An Application to the Dress Sector.

41. Osorio, J. (2016). El Tratamiento de la Incertidumbre en la Macroeconomía. Revista de Economía San Marcos, 2(1): 001-040.

42. Petroll, M. D. L. M., Damacena, C., \& Hernani, M. (2012). Medición y determinantes del valor de marca en la perspectiva del consumidor. Contabilidad y negocios, 3(6): 19-37.

43. Pinilla, V. (2009). Modelos Probabilísticos comunes. México: Facultad de Ingeniería, UNAM.

44. Riquelme Picart, I. B. (2016). Estimación del potencial de compra de los clientes de un proveedor peruano de productos de consumo masivo.

45. Rivas, J. A. (1981). Modelos estocásticos de elección de marca: Un contraste de utilidad para la representación del comportamiento del consumidor. Esic market, (35): 169-176.

46. Sánchez, V., \& Hasbleidy, Z. (2014). Modelos y configuraciones de cadenas de suministro en productos perecederos. Ingenieria y desarrollo, 32(1).

47. Téllez-León, I. E., \& Venegas-Martínez, F. (2016). Decisiones de consumo y portafolio con Utilidad Diferencial Recursiva Estocástica (UDRE): Modelos alternativos. EconoQuantum, 13(2): 51.

48. Thierauf, R. G. (1972). Toma de decisiones por medio de investigación de operaciones. Mexico: Limusa-Wiley.

49. Tobón, S., \& Pérez Acosta, A. M. (2016). El papel de la discriminación de estímulos en la lealtad hacia un producto de consumo masivo. Universidad \& Empresa, 18(31).

50. Velázquez, E. V., Farrera, M. Á. P., \& Cortazar, A. C. (2017). El análisis de la comunidad. Lacandonia, 2(1): 131-140.

51. Valbuena, F. A. B. (2016). Una evaluación de una marca global en la percepción del comportamiento de compra del consumidor: grupo Virgin. Revista Kairós Gerencial, 1(1), 17.

52. Vidal Holguín, C. J., Londoño Ortega, J. C., \& Contreras Rengifo, F. (2011). Aplicación de modelos de inventarios en una cadena de abastecimiento de productos de consumo masivo con una bodega y $\mathrm{N}$ puntos de venta, bibliotecadigital.univalle.edu.co

53. Yunda Padilla, E. G. (2009). Optimización estocástica del portafolio de compras de electricidad para distribuidoras en el Mercado Eléctrico Ecuatoriano (Doctoral dissertation, Quito: EPN, 2009).

54. Zhang, T., Siebers, P. O., \& Aickelin, U. (2016). Simulating user learning in authoritative technology adoption: An agent based model for council-led smart meter deployment planning in the UK. Technological Forecasting and Social Change, 106, 74-84. 